Recommendations Education programme to raise awareness of complications of pre-septal/orbital cellulitis.

- Involve opthalmology and otolaryngology at the earliest.

- Twice daily assessment of colour vision, eye movements and pupil reflexes, for early identification of complications.

- Ensure adequate analgesia.

- Re-audit, preferably with the use of one antibiotic combination, also looking at indications for changing from IV to oral along with total duration.

\section{C-REACTIVE PROTEIN, LEUKOCYTES AND ESR AS IDENTIFIERS OF INVASIVE BACTERIAL INFECTIONS}

doi:10.1136/archdischild-2012-302724.0950

${ }^{1} \mathrm{C}$ Zavarache, 1,20 Falup-Pecurariu. 'Department of Pediatrics, University Children's Hospital; '2Department of Pediatrics, Faculty of Medicine, Transilvania University, Brasov, Romania

Background C-reactive protein (CRP), erythrocyte sedimentation rate (ESR) and leukocyte count are used in several studies, for evaluation of invasive bacterial infections.

Aim Aim of the study to evaluate the usefulness of leukocytes, CRP and ESR as identifiers of invasive bacterial infections at children.

Methods We have prospectively evaluated 705 patients divided into two groups: 1) group A with 110 patients having invasive bacterial infections and 2) group $B$ with localized bacterial infections comprised of 595 patients.

Results Mean values for leucocytes was $12536 \pm 6642 / \mathrm{mm}^{3}$ for invasive bacterial infections versus $11263 \pm 5285 / \mathrm{mm}^{3}$ for localized bacterial infections $(p<0.05)$.

Mean ESR for invasive infections was $31,13 \pm 29,11 \mathrm{~mm} / \mathrm{hr}$ versus $25,94 \pm 22,93 \mathrm{~mm} / \mathrm{hr}$ for localized infections ( $<<0.08$ ). Mean CRP for invasive infections was $42,57 \pm 25,45 \mathrm{mg} / \mathrm{dl}$ versus $24.10 \pm 13.58 \mathrm{mg} / \mathrm{dl}(\mathrm{p}<0.04)$ for localized bacterial one.

Conclusion This study suggests that CRP, ESR and leucocytes are good indicators in diagnose of invasive bacterial infections.

\section{RECURRENT PRIAPISM PRESENTING WITH A RARE CAUSE IN AN INFANT}

doi:10.1136/archdischild-2012-302724.0951

C Warren, T Fontaine, S Bedwani. Child Health Department, Royal Cornwall Hospital, Truro, UK

Discitis in children is an important infective cause of morbidity and often has an insidious presentation, which creates difficulty in making a prompt diagnosis and instigating appropriate treatment.

Priapism in the paediatric population is a rare urological emergency. It is most commonly associated with sickle cell disease and leukaemia, causing a vaso-occlusive priapism, and rarely with spinal shock. To the authors' knowledge, priapism secondary to discitis has not previously been described in the literature.

We present a case of a 12 month old Caucasian child presenting with a three-week history of regular episodes of seemingly painful, persistent erections. He was noted to have a reluctance to sit or walk. Clinical examination was unremarkable other than priapic episodes. Neurological and haematological causes were considered but initial investigations were normal.

An MRI of his spine, performed due to a later rise in erythrocyte sedimentation rate and a clinical deterioration with complete refusal to mobilise, demonstrated inflammatory changes at the L3/ L4 level with a seven-millimetre collection, thus indicating discitis. $\mathrm{He}$ was treated with intravenous antibiotics and after ten days made a full recovery with complete resolution of his symptoms.

This is the first reported case of discitis presenting as recurrent priapism in an infant. We feel that this case highlights the need to consider infective causes in children presenting with priapism, so that diagnosis and treatment of a significant underlying cause is not delayed. Early spinal imaging may be worthwhile in cases of unexplained priapism, particularly when haematological causes have been excluded.

\section{ENCEPHALITIS DUE INFLUENZA A (H1N1) IN A SEVEN YEAR-OLD GIRL WITH GOOD RESPONSE TO OSELTAMIVIR}

doi:10.1136/archdischild-2012-302724.0952

'F Abbasi, ${ }^{2} \mathrm{D}$ Yadegarynia, ${ }^{3} \mathrm{~S}$ Korooni, ${ }^{2} \mathrm{M}$ Razavi. ${ }^{1}$ Bushehr University of Medical Sciences, Bushehr; IInfectious Diseases and Tropical Medicine Research Center, Shaheed Beheshti Medical University, Tehran; ${ }^{3}$ Shiraz University of Medical Sciences, Shiraz, Iran

Background and Aims Human infection with the novel H1N1 influenza virus was first reported in April 2009. Novel Influenza A (H1N1) virus produces higher mortality in young people. Different clinical manifestation of Influenza A (H1N1) has been reported. We present encephalitis due influenza A (H1N1) with good response to oseltamivir

Patient The patient was a seven year-old girl presented with mood change and gait ataxia from 5 days before admission. She also had fever, delusion, and lethargy. She had history of common cold several days before admission. She was treated with acyclovir with impression of encephalitis without improvement. In physical examination $(\mathrm{P} / \mathrm{E})$ she was febrile, there was no nuchal rigidity. $\mathrm{P} / \mathrm{E}$ of chest, abdomen and extremities were normal. Lumbar puncture was performed. Cerebrospinal fluid (CSF) was normal. CSF culture showed no growth after 48h. CBC, FBS, BUN, Cr, Na, K, ALT, AST, CRP and procalcitonin were all normal. HSV PCR was negative. Elecroencephalography (EEG) was performed that suggested encephalitis. Brain MRI was normal. Throat culture was obtained for the diagnosis of influenza A (H1N1) that was positive. The patient was treated with oseltamivir. The patient recovered after treatment and tests for equilibrium became normal.

Conclusions Encephalitis due to influenza A (H1N1) should be considered in every patient with signs and symptoms of encephalitis during influenza $\mathrm{A}(\mathrm{H} 1 \mathrm{~N} 1)$ pandemy.

\section{MILIARY TUBERCULOSIS IN AN IMMUNOCOMPETENT CHILD}

doi:10.1136/archdischild-2012-302724.0953

'CSG Garcez, 'S Carvalho, 'S Martins, ${ }^{1} T$ Pontes, ${ }^{1} \mathrm{~A}$ Antunes, ${ }^{1} \mathrm{~A}$ Gonçalves, ${ }^{1} \mathrm{C}$ Moreira, 1,2H Antunes. 'Pediatrics, Braga Hospital, Braga; 'Life and Health Sciences Research Institute, School of Health Sciences, University of Minho ICVS/3B's - PT Government Associate Laboratory, Braga/Guimarães, Portugal

Background Miliary tuberculosis (MT) refers to clinical disease resulting from lymphohematogenous dissemination of Mycobacterium tuberculosis. It affects primarily younger and immunocompromised children. Clinical presentation is highly variable and multiorgan involvement is common.

Clinical Case A nine-year Gypsy girl with a mild development delay and a history of epilepsy was presented to our hospital with a 19-day history of fever. She had completed two antibiotic treatments for suspected tonsillitis. Two years before she had had meningococcal meningitis. Family history was irrelevant. On admission, physical examination was normal and it was performed a chest radiography which revealed diffuse, bilateral, small lung nodules (Panel A). Ophthalmology diagnosed tubercular choroiditis in the right eye. An acute MT diagnosis was made. Lumbar puncture was normal and cranial magnetic resonance imaging showed several small nodular lesions - tuberculomas. Mantoux test: $14 \mathrm{~mm}$. In gastric aspirate cultures grew Mycobacterium tuberculosis without antibiotic resistance. Immune deficiency was excluded. She received 